Monatsschrift f. Geburtshülfe u. Gynäkologie 1936;102:I-IV

\title{
Contents, Vol. 102, 1936
}

Inhaltsverzeichnis.

Originalarbeite $\pi . \quad$ Seit $\theta$

Baudler, Udo, Gonadotrope Wirkstoffe im Urin von Tumorkranken. . 156 Baumgart, Elfríede, Zur Frage de $\Gamma$ Radikaloperationen bei chronischer

Adnexerkrankung 149

Bittmann, 0., Zur Berechtigungsfrage der Lumbalanästhesie in de $\Gamma$

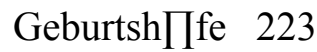

Bonilla, Francisco, undFritzGoebel, Über die Behandlung der Eklamp-

sie mit Thyroxin 193

Duseberg, Geo, Entstehung und Arten der Bauchhöhlenschwangerschaft 30

Dyroff, Rudolf, Erfahrungen an den ersten 100 gesetzlichen Sterilisierungen 9 Effkemann, Georg, Beitrag zum intermediären Fettstoîfwechsel bei Gestationstoxikosen(Blutfettsäuregehalt) 161

Ehrhardt, Karl, und Heinz Fischer-Wasels, Untersuchungen über den

Gehalt der menschlichen Plazenta an Corpus-luteum-Hormon . 80 Eufinger, H., und H. Molz, Elektrokardiographische Untersuchungen bei der Präeklampsic und Eklampsie 17

Fischer-Wasels, Heinz, siehe Ehrhardt, Karl

Föderl, Viktor, Untersuchungen über die durchschnittliche Geburtsdauer bei Erst-, Zweit- und Mehrgraviden und ihre Beziehungen zum Alter der Gebärenden 65

Fraymann, S. A., Die Geburt in Knieellenbogenlage (ohne Dammschutz) 324

Ganewskaja, R. S., und Erich Werbatus, Die Behandlung der nicht komplizierten weiblichen Gonorrhoe mit Ammoniaksilbersalzlösungen 52

Goebel, Fritz, siehe Bonilla, Francisco.

Hollósi, Karl, Intrakranielle Blutungen bei Neugeborenen .... 317

Kramm, H., Über Thymus persistens und Entbindung 311

Lindner, Gerda, Zur Frage der konservativen Behandlung oder Operation der chroniseh entzündlichen Adnexe 216 Molz, H., siehe Eufinger, $\mathrm{H}$.

Mükerrem, Nuri, Zur Frage der Spätschädigungen des Gefäßsystems nach Eklampsie und Präeklampsie 282

Mueller, Ernst Adolf, Der „biologische Arzt” als Gynäkologe und

Geburtshelfer 58

Nuri siehe Mükerrem. 
Polonsky, J. N., Die unmittelbaren und entfernten Resultate des

Kaiserschnitts 43

Rossenbeck, H., 1st die physiologische Schwangerschafts-Azidose

ketogener Natur? 129

]/y Inhaltsverzeichnis.

Seit $\theta$

Schulze, Eberhard, Das Scleroedeina neonatorum und seine Behand-

lung mit Thyroxin (Hierzu Tafel I) 303

Schwalm, Horst, Mütterîursorge und Erbgesundïieit 93

Seiíz, L., Vollkoinmener Zyklus und unvollkommener Zykius, echte

Menstruation und Pseudomenstruation .

Sigwart, W·, Die Veiitrosuspension des Uterus und ihre Dauerresultate 286 Snoo, K. De, Die

Ekiampsie und die vorzeitige Lösung der Plazenta 257 Späih, Berthold, Untersuchungen über die

Veränderung des Blut-

cholingehaltes in der normalen und toxischen Sehwangerschaft 167 Tschertok, R. A., Zur

Diagnose und Therapie der Amenorrhoe . . 202 Werbatus, Erich, siehe Ganewskaja, R. S.

Winkler, Hellmuth, Über eine seltene Form der wahren, diîfusen

Mammahypertrophie 144

Wirth, L., Naevus teleangiektaticus und Sehwangerschaft 298

Wlassow, W. N., Zur Diagnose der ausgetragenen resp. fast aus-

getragenen ektopischen Gravidität 22

Grenzgebiete der Geburtshilfe und Gynäkologie.

Der Stofînechsel bei Schwangeren. (VI. Jahresübersicht.) Von Dr.

phil. et med. O. Mühlbock- Berlin 99

Physikalische Heilmethoden einschließlich der Hydrotherapie. Von

Prof. Dr. Hermann F r a n k e n - Freiburg i. Br 184

Die Erkrankungen der Leber, der Niere und der ableitenden Harnwege

in ihren Beziehungen zur Geburtshilfe und Gynäkologie. Von

Hans Rupp- Bonn 242

Kreislaufbericht über das Jahr 1935. Von H. Reichenmiller-

Tübingen 329

Sitzungsberichte aus geburtshilflich -gynäkologischen Gesellschafíen.

Gynäkologische Gesellschait zu Breslau. Sitzung vom 15. Nov. 1935

Gesellschaît für Geburtshilfe und Gynäkologie zu Berlin. Sitzung

vom 22. November $1935 \quad 118$

-, Sitzung vom 10. Januar $1936 \quad 351$

Geburtshilîlich-Gynäkologische Gesellschaît in Wien. Sitzung vom

14. Januar $1936 \quad 121$

-, Sitzung vom 11. Februar $1936 \quad 190$

-, Sitzung vom 10. März 1936

-, Sitzung vom 12. Mai $1936 \quad 348$

Mittelrheinische Gesellschaît îür Geburtshilîe und Gynäkologie.

Sitzung in Frankfurt a. Main am 16. Februar 1936 .... 353

Deutsche Gesellschaît iür Gynäkologie. 24. Tagung vom 23. bis

26. Oktober in München (Schluß) 107

Buchbesprechungen 123, 363 
Hugo Sellheim $\ddagger \quad$ 192, 253

Hochschul- und Tagesnachrichten $\quad 128,256,364$ 